\title{
Participação popular no controle da hanseníase: um desafio para o serviço de saúde
}

\author{
Social participation in leprosy control: a challenge for health services \\ Participación popular en el control de la lepra: un desafío para el servicio de salud
}

\author{
Fabiana Nascimento Lopes ${ }^{I}$ Francisco Carlos Félix Lana ${ }^{I I}$
}

\begin{abstract}
RESUMO: O objetivo do estudo foi analisar, na perspectiva de conselheiros de saúde e líderes comunitários, o processo de intervenção do serviço de saúde no controle da hanseníase. Trata-se de uma pesquisa de abordagem qualitativa, realizada em agosto de 2013, no município de Almenara, Minas Gerais. Os dados foram coletados por meio de entrevista semiestruturada com 10 conselheiros de saúde e sete líderes comunitários. O tratamento dos dados foi realizado a partir da análise qualitativa proposta por Laville e Dione. Os líderes comunitários e os conselheiros de saúde não identificam as ações realizadas pelo serviço de saúde relacionadas à hanseníase no município e não participam, individualmente ou como grupo, das atividades de controle da doença. Conclui-se que o serviço de saúde de Almenara não está sendo capaz de promover a participação social no controle dessa doença, conforme estabelecido pelo Programa Nacional de Controle da Hanseníase.

Palavras-Chave: Hanseníase; prevenção \& controle; educação em saúde; participação comunitária.
\end{abstract}

ABSTRACT: The aim of this qualitative study was to examine the process of health service intervention in leprosy control as seen by local health policy council members and community leaders. The study was conducted in August 2013 in the municipality of Almenara, Minas Gerais, Brazil. Data were collected from 10 health policy counsellors and seven community leaders using a semi-structured questionnaire. Data were analysed according to Laville and Dione's qualitative approach. Neither the community leaders nor the health policy counsellors identified leprosy-related measures by health services in the municipality, and took no part, individually or as a group, in leprosy control activities. It was concluded that health services in Almenara are not capable of promoting social participation in leprosy control as required by the National Leprosy Control Programme. Keywords: Leprosy; prevention \& control; health education; consumer participation.

RESUMEN: El objetivo del estudio fue analizar desde la perspectiva de los consejeros de salud y líderes comunitarios, el proceso de intervención del servicio de salud en el control de la lepra. Se trata de una investigación de abordaje cualitativo, realizada en agosto de 2013, en el municipio de Almenara, Minas Gerais, Brasil. Se han recolectado los datos a través de entrevistas semiestructuradas con 10 consejeros de salud y siete líderes comunitarios. Se han analizado los datos por medio de análisis cualitativo propuesto por Laville y Dione. Los líderes comunitarios y consejeros de salud no identifican las medidas adoptadas por el servicio de salud en relación a la lepra en el distrito y no participan, de forma individual o como un grupo, de las actividades de control de la enfermedad. Se concluye que el servicio de salud de Almenara no es capaz de promover la participación social en el control de la lepra, según lo establecido por el Programa Nacional de Control de Lepra.

Palabras Claves: Lepra; prevención \& control; educación en salud; participación comunitaria.

\section{INTRODUÇÃO}

O Programa Nacional de Controle da Hanseníase (PNCH) estabelece que as práticas de educação em saúde, no controle da doença, devem estar em consonância com a Política Nacional de Promoção da Saúde, considerando a busca por um atendimento integral, o estímulo ao autoexame e à investigação dos contatos domiciliares, bem como a orientação e o apoio para autocuidado, prevenção e tratamento das incapacidades físicas e suporte psicológico ${ }^{1}$. Essas atividades são de responsabilidade das três esferas de governo, que deverão buscar parcerias com as instituições e as entidades da sociedade civil para a construção e a divulgação de conhecimentos sobre a hanseníase e o desenvolvimento de uma rede de atenção integral ao doente e à sua família ${ }^{1}$.

Nessa perspectiva, ao se pensar em um projeto de educação em saúde, deve-se ter em mente não apenas a construção de uma consciência sanitária capaz de reverter o quadro de saúde da população, mas ter como proposta a intensificação da participação popular em uma visão mais democratizante das políticas públicas².

${ }^{I}$ Mestre em Enfermagem, pelo Programa de Pós-Graduação em Enfermagem da Escola de Enfermagem da Universidade Federal de Minas Gerais. Membro do Núcleo de Estudos e Pesquisa em Hanseníase. Belo Horizonte, Minas Gerais, Brasil. E-mail: fabiana.nlopes@yahoo.com.br.

IDoutor em Enfermagem. Professor Associado IV do Departamento de Enfermagem Materno-Infantil e Saúde Pública da Escola de Enfermagem da Universidade Federal de Minas Gerais. Coordenador do Núcleo de Estudo e Pesquisa em Hanseníase. Belo Horizonte, Minas Gerais, Brasil. E-mail: xicolana@ufmg.br. 
O PNCH estabelece que as ações de educação em saúde sejam realizadas de forma integrada a mobilização social, com a proposta de envolver os diferentes atores sociais na sua elaboração, excussão e avaliação ${ }^{1}$. No caso da hanseníase, observa-se que a comunicação estabelecida pelo $\mathrm{PNCH}$ mantém-se verticalizada e fragmentada, com o predomínio de campanhas que centralizam suas ações em materiais educativos, em detrimento de atividades participativas e horizontalizadas. Isso favorece a existência de uma lacuna entre a produção e a institucionalização de um novo modo de intervir na doença e de discutir sobre os aspectos que a caracterizam ${ }^{3}$.

Nesse contexto, o presente estudo teve como objetivo analisar, na perspectiva de conselheiros de saúde e líderes comunitários, o processo de intervenção do serviço de saúde no controle da hanseníase.

Este artigo faz parte de um projeto maior intitulado Perspectivas dos sujeitos envolvidos em movimentos sociais sobre a hanseníase e seu controle em uma área endêmica, desenvolvido pelo Núcleo de Estudo e Pesquisa em Hanseníase (NEPHANS), da Escola de Enfermagem da Universidade Federal de Minas Gerais.

\section{ReVISÃo DE LITERATURA}

$\mathrm{O} \mathrm{PNCH}$ tem como objetivo orientar as práticas dos serviços de saúde em todos os diferentes níveis de atenção, fortalecendo as ações de vigilância epidemiológica, a promoção da saúde, com base na educação permanente e na assistência integral aos portadores da doença ${ }^{1}$.

A descentralização das ações em saúde para atenção básica $(\mathrm{AB})$ possibilitou maior acesso aos serviços de saúde por parte da população. A mudança do modelo assistencial trouxe novas possibilidades para a atenção à saúde, principalmente relacionadas à mudança no paradigma assistencial, caracterizada pela substituição de um modelo baseado em especialidades médicas para um modelo centralizado na vigilância em saúde, com ênfase no sujeito e na família 4 .

A realização de uma assistência integral ao portador da hanseníase requer a organização de equipes multidisciplinares no Sistema Único de Saúde, na atenção básica, na média e alta complexidade, para que possam atender cada caso dentro de suas necessidades e dos princípios da equidade e da integralidade ${ }^{5}$.

A democratização das ações da $\mathrm{AB}$ passa pela participação e pelo envolvimento da sociedade no controle das doenças que afetam a população. A formação de grupos sociais organizados em torno de seus ideais tem o poder de gerar mudanças essenciais para a construção de um bem-estar social fundamentado em um coletivo consciente e mobilizado ${ }^{6}$.

Atualmente, observa-se uma ausência de cidadãos no cenário político, também percebida na prática dos serviços de saúde. Isso pode denunciar a presença de resquícios de um modelo de saúde embasado em ações normativas e discursos coercitivos ${ }^{6,7}$, de um passado de opressão que moldou a participação e que ainda se mantém nos dias atuais, contribuindo para uma participação restrita da população $0^{7,8}$.

\section{Metodologia}

Neste estudo, optou-se pela utilização da abordagem qualitativa, por se revestir dos significados e da intencionalidade como essenciais aos atos, às relações e às estruturas sociais?

O estudo foi desenvolvido na cidade de Almenara, localizada no nordeste de Minas Gerais, que, juntamente a outros municípios dos estados de Minas Gerais, Espírito Santo e Bahia, faz parte do cluster ${ }^{6}$. Elegeram-se como sujeitos do estudo conselheiros municipais de saúde (CMS) e líderes comunitários (LC), por serem pessoas da comunidade que ocupam uma posição estratégica dentro do município.

A eleição dos sujeitos de pesquisa teve como critério de inclusão pertencer ao Conselho Municipal de Saúde e/ou a um movimento social no município de Almenara. Para a coleta de dados, foram convidados os 11 titulares do CMS, e os oito LC; isto é, o universo de conselheiros atuais do CMS e de instituições cadastradas no município. Os LC foram identificados a partir do cadastro das instituições sociais e o convite para participação foi destinado ao presidente da instituição. Dessa forma, foram eleitos para o estudo 19 sujeitos e participaram 17, sendo sete LC e 10 CMS. Ocorreu a recusa de um líder comunitário e de um conselheiro de saúde, que não estava no município, no momento da coleta de dados.

A coleta dos dados foi realizada pela própria pesquisadora, por meio de roteiro de entrevista semiestruturado. As entrevistas foram gravadas com o consentimento do participante e, logo após, foram transcritas. A fim de assegurar o anonimato, os sujeitos foram identificados de acordo com o movimento social a que pertenciam e com a ordem em que foram realizadas as entrevistas, como por exemplo: CMS-1 (conselheiro municipal de saúde-1); LC - 1 (líder comunitário-1).

Os dados obtidos neste estudo foram tratados conforme a análise qualitativa proposta por Laville e Dionne $^{10}$. O tratamento dos dados possibilitou a construção de duas unidades de análise: $\mathrm{O}$ serviço de saúde e a hanseníase: o que eles estão fazendo? e Participação popular no controle da hanseníase: um desafio.

\section{Resultados e Discussão}

Os resultados são apresentados e discutidos segundo as duas unidades de análise emergentes do estudo e destacadas a seguir. 


\section{O serviço de saúde e a hanseníase: o que eles estão fazendo?}

As ações do PNCH no município de Almenara foram descentralizadas para a $\mathrm{AB}$ a partir de 2005. $\mathrm{O}$ município apresenta um modelo de atenção à saúde em hanseníase que mescla o modelo descentralizado, que atende à população coberta pela estratégia de saúde da família (ESF), e o centralizado, praticado pelo centro de especialidades médicas, que é a referência municipal para a atenção à hanseníase e atende à parcela da população que não é coberta pela ESF (aproximadamente 35\%) ${ }^{11}$.

Certos sujeitos do estudo, ao serem questionados sobre o trabalho que o serviço de saúde tem realizado com relação à hanseníase, responderam que não teriam como abordar a temática, por não saberem como o serviço tem trabalhado a doença no município. Entre esses, encontram-se conselheiros municipais de saúde.

Os entrevistados, ao descreverem o trabalho desenvolvido pelo serviço de saúde, ao invés de abordarem as ações realizadas, apontaram fragilidades, como a alta rotatividade e a falta de interesse dos profissionais, o atraso no diagnóstico e a dificuldade em conseguir recursos financeiros.

Eu acho que aqui em Almenara [...] falta médico e os que tem, não gosta de ver essa doença, é um trabalho ainda muito fraco, precisa ser um trabalho voltado realmente para a questão do paciente, da doença, a prevenção, a orientação e a informação nas comunidades, porque falta muito isso ainda. (LC-3)

A existência de fragilidades na integração das ações do $\mathrm{PNCH}$ na $\mathrm{AB}$, geralmente, não decorre das propostas estabelecidas pelo Ministério da Saúde, mas da insuficiência da gestão municipal na ESF, observada em relações de trabalho precárias, vínculos empregatícios frágeis e dificuldade de locação do profissional médico, entre outros aspectos ${ }^{12,13}$.

Para outros, o engajamento no controle da hanseníase passa pela vontade dos profissionais de saúde de trabalhar a doença junto à população, porque, muitas vezes, a enfermidade não é considerada prioritária para o serviço de saúde.

Eu acho que hoje eles se preocupam mais com outras coisas, eles não se preocupam se a pessoa tem ou não hanseníase, se a pessoa vai fazer um diagnóstico, um exame de hanseníase ou não vai; eles se preocupam mais, hoje, é com a pressão, o problema cardíaco[...] (LC-1)

Há necessidade de maior comprometimento político dos gestores no estabelecimento das ações de controle da hanseníase, considerando-a como um problema de saúde prioritário no município. Quando a gestão toma a decisão de priorizá-la e enfrentá-la, o cenário da doença pode mudar, melhorando os índices de detecção, tratamento precoce e participação da população nas ações desenvolvidas ${ }^{14}$. A ausência de priorização de determinadas doenças retrata uma parte da crise do setor de saúde no Brasil, em que se evidencia um desrespeito aos direitos dos cidadãos ${ }^{15}$. Entretanto, esse fato não deve servir de justificativa para um atendimento de baixa qualidade decorrente da falta de compromisso dos profissionais com os usuários ${ }^{14,15}$.

As principais ações reconhecidas pelos entrevistados são as que dão maior visibilidade ao tema, como o uso de cartazes e as campanhas de divulgação, ao passo que o diagnóstico, o tratamento, o acompanhamento dos doentes, ações de menor visibilidade, não foram identificados.

Não, o que eu vejo são muitos cartazinhos nos postos de saúde [...] que falam da doença, o que é, como identificar [...] o que a gente mais vê são os cartazes, agora esse tipo de programa passeata, carreata, anuncio em televisão, rádio, nada disso eu vejo. (CMS-10)

A conexão, estabelecida pelos participantes, entre as ações realizadas pelo serviço de saúde e o uso de cartazes e panfletos pode ser justificada pela ampla utilização desses recursos na divulgação da doença ${ }^{16}$. Os cartazes e os panfletos possuem um caráter educativo, principalmente na relação entre profissionais de saúde e população, mas, muitas vezes, são utilizados de forma instrumental e verticalizada junto às pessoas, sem que haja interação entre as partes ${ }^{16,17}$.

A utilização dos cartazes e dos panfletos como forma de divulgar a doença e de levar informação à população foi avaliada pelos participantes como insuficiente, porque grande parte das pessoas que frequentam as unidades de saúde não tem o hábito de ler e possui baixa escolaridade.

Eu avalio de maneira ineficiente. Porque a forma de mostrar a doença para as pessoas é uma maneira só visual, eu vejo [...] e tenho que ler para ter a informação e nós não temos uma sociedade que tem cultura, que tem o hábito de leitura [...] temos uma sociedade pobre, que tem pouca e o mau hábito de leitura, ele entra no posto de saúde, ele não vai ler cartaz [...] dessa forma não consegue atingir, não consegue informar [...] (CMS -10)

A utilização de cartazes é compreendida como parte da cultura dos serviços de saúde, por promoverem a mobilização do público, mas se sabe que nem sempre são lidos pelas pessoas que frequentam as unidades ${ }^{17}$. As ações educativas podem contribuir para o desenvolvimento de conhecimento e o combate à desinformação sobre a hanseníase ${ }^{18,19}$. Quando realizadas em pequenos grupos, possibilitam a interação, a reflexão e a construção de novos saberes ${ }^{18}$. $\mathrm{O}$ cuidado em saúde deve ser construído na relação entre profissionais de saúde, família e doentes, na troca de informações, como forma de propor a melhoria do estado de saúde e da qualidade de vida de todos ${ }^{20}$.

A dificuldade no desenvolvimento de ações de prevenção, divulgação e controle da hanseníase no dia 
a dia das unidades de saúde e na comunidade fica mais evidente quando os participantes apontam para a necessidade de trabalhar a doença dentro dos serviços, de identificar as regiões prioritárias para o seu controle no município e de envolver mais os profissionais de saúde e as instituições sociais, ações já previstas no $\mathrm{PNCH}$.

[... ]então eu acho que poderíamos trabalhar mais em prevenção e descobrir as áreas da cidade onde tem a maior concentração e ver se isso tem alguma relação, se realmente a gente consegue neutralizar a doença em pontos estratégicos da cidade[...] (CMS-10)

O sistema de saúde vigente no país ainda é incapaz de promover maior interação com seu ambiente social ${ }^{6,7}$. A busca pela compreensão das reais necessidades de saúde da população e pelo conhecimento do seu perfil de (re)produção social auxilia na compreensão de como ocorre a inserção dos sujeitos no seu contexto social e possibilita evidenciar as suas potencialidades de saúde e de sobrevivência ${ }^{18}$.

No caso da hanseníase, os principais desafios no controle da doença visam manter a qualidade dos serviços e assegurar que todas as pessoas acometidas pela doença, independentemente de onde vivam, tenham igual oportunidade de serem diagnosticadas e tratadas por profissionais de saúde competentes ${ }^{21}$, que trabalhem não apenas os aspectos anatopatológico, mas também as repercussões psicossociais da doença ${ }^{22}$.

As dificuldades, que o serviço de saúde de Almenara tem enfrentado no controle da doença, contribuíram para que o retorno da centralização no atendimento dos casos de hanseníase seja considerado, pelos entrevistados, como uma estratégia capaz de melhorar o acesso ao diagnóstico, ao tratamento e ao atendimento aos familiares.

Eu acredito que o trabalho com hanseníase tinha que estar pautado na prevenção [...], deveria ter um polo dentro da comunidade, onde todas as pessoas passariam por ele, ficaria nesse polo e receberia orientação, capacitação [...] convidaria dermatologista ou uma pessoa que fosse especialista [... ] e trouxesse para o município, para trabalhar dentro da comunidade [...] (LC-3)

Esses aspectos apontam para a necessidade de estratégias que fortaleçam a $\mathrm{AB}$ no controle da hanseníase $^{23}$. A descentralização da assistência dos casos para a $A B$ deve ser vista como uma ação importante, porém não a única, uma vez que os fatores regionais, culturais, educacionais, socioeconômicos, geográficos e políticos devem ser considerados na elaboração das estratégias ${ }^{1,24}$. As ações de diagnóstico, tratamento e controle de comunicantes devem ocorrer na $\mathrm{AB}$, mas o acompanhamento das intercorrências, bem como o tratamento, a prevenção e a reabilitação das incapacidades devem permanecer centralizados ${ }^{24}$.

Foi possível observar as dificuldades e as fragilidades encontradas para que as ações de descen- tralização do atendimento aos casos de hanseníase ocorram de fato. Acredita-se-que, para vencer esses obstáculos, além de vontade política, é necessário o envolvimento dos profissionais de saúde, dos gestores, da comunidade e dos movimentos sociais nas ações propostas pelo $\mathrm{PNCH}$.

\section{O desafio da participação popular no controle da hanseníase}

O controle social configura-se como uma das formas de participação popular na saúde. Refere-se à participação direta da comunidade no processo de gestão pública, em que a população apropria-se de meios e instrumentos para planejar, fiscalizar e analisar as ações e os serviços ${ }^{21}$. $O$ controle social é resultante do processo de consolidação da democracia, o que faz com que seu fortalecimento contribua para o alargamento da esfera pública ${ }^{25,26}$. Nessa perspectiva, a execução do controle social está condicionada à existência de uma democracia participativa e ao estabelecimento de canais de participação ${ }^{26}$.

A participação social não tem sido exercitada ou estimulada de forma sustentada e consequente no país, mas é importante buscar superar os obstáculos que dificultam a efetiva participação e ressaltar os avanços que ocorreram como forma de viabilizar as possíveis transformações na política de saúde, considerando os usuários do serviço de saúde como sujeitos diante do compromisso e da responsabilidade de promover mudanças ${ }^{6}$.

A maior parte dos participantes do estudo, ao ser questionada sobre a existência de discussões acerca da hanseníase nos movimentos sociais de que participa, disse que a doença nunca foi pauta de discussão. As justificativas atribuídas à falta de discussão são o não envolvimento com a causa específica da hanseníase, a abordagem da saúde como um todo, o foco nos assuntos da categoria, entre outras.

Não, porque a gente luta pela saúde como um todo, não por situações ou por doença, nosso papel é lutar por saúde[...] (CMS-4)

Não, porque a gente vê mais as coisas da categoria mesmo, tipo as questões salariais [...] (LC-7)

A falta do engajamento político da sociedade e a ausência de uma ação política que envolva mais os cidadãos são evidências do esvaziamento da democracia idealizada ${ }^{7,26}$, refletidas na Constituição Cidadã ${ }^{26}$. Sendo assim, deve-se buscar meios capazes de envolver a comunidade e, principalmente, os movimentos sociais no controle da hanseníase e de outras doenças, em que a vontade de participar seja concretizada em ações.

A respeito das ações de combate à hanseníase, também relataram que os movimentos sociais de que participam nunca realizaram atividades de divulgação ou controle da doença, exceto o representante da Pastoral da Criança. 
Não, não vou falar que realizou e nem participou, porque não teve. (CMS-5)

O mesmo foi verificado quando os sujeitos foram questionados se individualmente já participaram de alguma atividade relacionada à hanseníase. Grande parte disse que não, exceto os profissionais de saúde presentes no estudo e o representante da Pastoral da Criança.

Não, porque nunca fui convidada e nunca nem fiquei sabendo. (LC-2)

O desenvolvimento de práticas democráticas eficazes pode fortalecer o controle social, mas cabe ressaltar que a participação não é um conteúdo que pode ser transmitido, como também não se pode adquiri-la por um simples treinamento. É resultado de uma mentalidade e de um comportamento a serem construídos pela reflexão crítica e pelo amadurecimento do cidadão ${ }^{27}$. Mesmo com todo o empenho na consolidação de uma visão moderna de participação social, o quadro de reordenação social e política ainda não alcançou um sustentável avanço no que diz respeito aos direitos civis, políticos e sociais?

$\mathrm{O}$ envolvimento das pessoas em ações comunitárias depende mais do seu querer se envolver do que da realização de capacitações ${ }^{27}$, mas se acredita que o desenvolvimento de ações educativas seja capaz de sensibilizar as pessoas para o engajamento com as causas da comunidade.

A falta de empenho e até mesmo de compreensão da sua função como CMS fica mais evidente quando um dos conselheiros participantes justifica o seu não envolvimento com as ações de saúde por se considerar um conselheiro interno, que não tem atividades fora das reuniões.

Também não, nós sempre fomos conselheiro interno [...] o que mais nós tínhamos vontade, era sair, ter a liberdade, é fiscalizar, visitar, mas nunca tivemos esse cartaz, nós atuamos mais é na área de aprovar [...] mas na hora de você querer trabalhar junto para poder resolver esses problemas você não é ninguém [...] (CMS - 2)

A fala do participante aponta para uma visão equivocada de sua função como conselheiro de saúde. Para que as diretrizes propostas pela Resolução n³33/03 (que aborda as competências dos conselhos de saúde) sejam cumpridas pelos conselheiros, é necessário que tenham uma capacidade técnica para tal, o que, na maioria das vezes, não possuem, podendo comprometer a sua efetiva participação ${ }^{28}$.

Os conselhos representam importante instrumento cujo objetivo é romper com as tradicionais formas de gestão, ao fazer com que as decisões sejam tomadas de maneira democrática e transparente, por meio de processos participativos ${ }^{29}$. Mas, para que isso ocorra, é de fundamental importância que os consel- heiros tenham a consciência de suas responsabilidades perante a sociedade e que busquem melhorar sua atuação, pautada na ética e na cidadania ${ }^{29}$.

A atuação dos conselhos de saúde como espaço público e democrático é questionável, devido ao distanciamento que se observa entre os movimentos sociais, a população e a institucionalização do controle social, que, não raro, só existe no papel?

Assim como em outro estudo ${ }^{30}$, verificou-se que o controle da hanseníase, a partir das metas propostas pela Organização Mundial da Saúde, configura-se em um desafio para os serviços de saúde de lugares endêmicos. Esse desafio refere-se à organização dos serviços de saúde locais, à sua capacidade de mobilização e de enfrentamento das dificuldades impostas pela doença, quais sejam, longo período de incubação, discriminação, elevado número de casos, entre outras ${ }^{30}$.

\section{Conclusão}

Conclui-se que o serviço de saúde de Almenara não está sendo capaz de promover a participação social no controle da hanseníase, conforme estabelecido pelo PNCH. Os gestores e os profissionais de saúde têm apresentado dificuldades para trabalhar a doença na comunidade e para inserir as instituições sociais do município em seu controle. Os participantes do estudo deixaram claro que a participação social no controle da hanseníase não ocorre e que as estratégias utilizadas não são capazes de provocar mudanças na população.

Tornam-se necessários novos trabalhos que busquem investigar como o serviço de saúde de Almenara trabalha a informação e o conhecimento científico produzido sobre a hanseníase junto à população, a fim de melhor explicitar as falhas que podem ocorrer no processo de comunicação e divulgação da doença.É também importante o investimento em pesquisas que possam explorar as inter-relações entre a participação popular, a comunicação e a educação permanente nas ações de controle de hanseníase em áreas endêmicas.

A limitação deste estudo relaciona-se à localidade municipal e regional em que foi realizado, bem como ao número reduzido de participantes. Cabe ressaltar, entretanto, que seu delineamento possibilitou evidenciar o processo de intervenção empregado no município para o controle da hanseníase.

\section{REFERÊNCIAS}

1.Ministério da Saúde (Br). Secretaria de Vigilância em Saúde. Portaria n 3.125, de 07 de outubro de 2010. Brasília (DF): Gabinete Ministerial; 2010.

2.Gomes LB, Merhy EE. Compreendendo a educação popular em saúde: um estudo na literatura brasileira. Cad Saúde Pública. 2011; 27:7-18. 
3.Kelly-Santos A, Monteiro S, Rosember B. Significados e uso de materiais educativos sobre a hanseníase segundo profissionais de saúde pública do município do Rio de Janeiro, Brasil. Cad Saúde Pública. 2009; 25:857-67.

4.Santos AS, Castro DS, Falqueto A. Fatores de risco para a transmissão da hanseníase. Rev Bras Enferm. 2008; 61:738-43.

5.Palmeira I P, Queiroz ABA, Ferreira M A. Marcas em si: vivenciando a dor do (auto) preconceito. Rev Bras Enferm. 2013; 66:893-900.

6.Ribeiro F B, Nascimento, MAA. Exercício de cidadania nos conselhos locais de saúde: a (re) significação do "ser sujeito”. Rev baiana saúde pública. 2011; 35:152-66.

7.Silva CV, Silva DFL, Souza EM. A participação da sociedade civil na democratização do setor de saúde no Brasil. Rev bras educ méd. 2103; 37:254-9.

8.Vidal ECF, Saraiva KRO, Dodt RCM, Vieira NFC, Barroso MGTB. Democracia e participação cidadã: um debate sobre as práticas de educação em saúde. Rev Gaúcha Enferm. 2008; 29(3):475-80.

9.Minayo M C. O desafio do conhecimento. 12a ed. São Paulo: Hucitec; 2010.

10.Laville C, Dionne J. A construção do saber. Belo Horizonte (MG): Editora UFMG; 1999.

11.Lanza FM, Lana FCF. Decentralization of leprosy control actions in the micro-region of Almenara, State of Minas Gerais. Rev Latino-Am Enfermagem. 2011; 19:187-94.

12.Dias RC, Pedrazzani ES. Políticas públicas na hanseníase: contribuição na redução da exclusão social. Rev Bras Enferm. 2008; 61:753-6.

13.Brandão P. Assistência ao portador de hanseníase. Rev Bras de Enferm. 2008; 61:781-3.

14.Ximenes Neto FRG, Martins FR, Liberato BTG, Carvalho Filho JPC, Aguiar ERB, Martins AR. Ações de sustentabilidade para o controle da hanseníase: a experiência do município de Cariré - Ceará. Sanare (Sobral, Online). 2011; 10(2):71-4.

15.Veloso RC, Ferreira MA. Saúde e serviços: relações estabelecidas com os usuários à luz das representações sociais da cidadania. Rev enferm UERJ. 2013; 21:60-5 16.Santos AK, Monteiro SS, Ribeiro APG. Acervo de materiais educativos sobre hanseníase: um dispositivo da memória e das práticas comunicativas. Interface Comum, Saude, Educ. 2010; 14:37-51.

17.Santos AK, Ribeiro APG, Monteiro S. Hanseníase e práticas da comunicação: estudo de recepção de materiais educativos em um serviço de saúde no Rio de Janeiro. Interface - Comum, Saude, Educ. 2012; 16:205-18.
18.Berardinelli LMM, Figueredo TFL, Oliveira AS, Santos I, Giron MN, Ramos JP. Hipertensão arterial e conhecimento popular: potencializando o cuidado. Rev enferm UERJ. 2013; 21:446-51.

19.Moreira AJ, Naves JM, Fernandes LFRM, Castro SS, Walsh IAP. Ações educativas sobre hanseníase na população das unidades básicas de saúde de UberabaMG. Saúde debate. 2014; 38:234-43

20.Crispim JA, Fiorati RC, Queiroz AAR, Pinto IC, Palha PF, Arcêncio RA. Tuberculose no contexto das famílias: as vivências de familiares e pacientes acometidos pela doença. Rev enferm UERJ. 2013; 21:606-11.

21.Opromolla PA, Laurenti R. Hansen's disease control in the State of São Paulo: a historical analysis. Rev Saude Publica. 2011; 45:195-203.

22.Palmeira IP, Quiroz ABA, Ferreira MA. Marcas em si: vivenciando a dor do (auto) preconceito. Rev Bras Enferm. 2013; 66:893-900.

23.Lanza FM, Vieira NF, Oliveira MMC, Lana FCF. Instrumento para avaliação das ações de controle da hanseníase na Atenção Primária. Rev Bras Enferm. 2014; 67:339-46.

24.Arantes CK, Garcia MLR, Filipe MS, Nardi SMT, Paschoal VDA, Martins AR. Avaliação dos serviços de saúde em relação ao diagnóstico precoce da hanseníase. Epidemiol serv saúde. 2010; 19:155-64.

25.Cotta RMM, Martins PC, Batista RS, Franceschinni SCC, Priore SE, Mendes FF. O controle social em cena: refletindo sobre a participação popular no contexto dos conselhos de saúde. Physis (Rio J). 2011; 21:1121-37.

26.Gerschman S. Conselhos municipais de saúde; atuação e representação da comunidade populares. Cad Saúde Pública. 2004; 20:1670-81.

27.Cotta RMM, Cazal MM, Martins PC. Conselho Municipal de Saúde: (re) pensando a lacuna entre o formato institucional e o espaço de participação social. Ciênc saúde coletiva. 2010; 15:2437-45.

28.Santos SF, Vargas AMD, Lucas SD. Conselheiros e usuários do conselho municipal de saúde de Belo Horizonte: características sociais e representatividade. Saude soc. 2011; 20:483-95.

29.Rocha ACRP, Landim FLP, Caprara A, Lefèvre A, Lefèvre $\mathrm{F}$. $\mathrm{O}$ discurso coletivo de ex-hanseniano morador de um antigo leprosário no nordeste do Brasil. Interface - Comum, Saude, Educ. 2011; 15:213-23.

30.Silva AR, Santos ARR, Santos GMC, Silva VEB, Gonçalves EGR. Leprosy in Buriticupu, State Maranhão: actives search in the general population. Rev Soc Bras Med Trop. 2013; 45:199-202. 